\title{
Preparation of Ultrabright AIE Nanoprobes via Dynamic Bonds
}

Qing Wan ${ }^{\mathrm{a}}$, Chengbin $\mathrm{He}^{\mathrm{a}}$, Ke Wang ${ }^{\mathrm{b}}$, Meiying Liu ${ }^{\mathrm{a}}$, Hongye Huang ${ }^{\mathrm{a}}$, Qiang Huang ${ }^{\mathrm{a}}$, Fengjie Deng ${ }^{\mathrm{a},{ }^{*} \text {, }}$ Xiaoyong Zhang, ${ }^{\mathrm{a}, *}$ and Yen Wei $\mathrm{i}^{\mathrm{b}, *}$

a Department of Chemistry and Jiangxi Provincial Key Laboratory of New Energy Chemistry, Nanchang University, 999 Xuefu Avenue, Nanchang 330031, China

b Department of Chemistry and the Tsinghua Center for Frontier Polymer Research, Tsinghua University, Beijing, 100084, P. R. China.

Yen Wei, Ph.D.

Chair Professor of Chemistry and Director of The Center for Frontier Polymer Research, Department of Chemistry, Tsinghua University, Beijing 100084, China

Tel. +86-10-6277-2674; Fax: +86-10-6277-1149

Email: weiyen@tsinghua.edu.cn

(or Herman B. Wagner Professor of Chemistry

Department of Chemistry, Drexel University, Philadelphia, PA 19104, USA; weiyen@drexel.edu)

And

Xiaoyong Zhang, Ph.D.

Associate Professor of Chemistry

Department of Chemistry and Jiangxi Provincial Key Laboratory of New Energy Chemistry, Nanchang University,

Nanchang 330031, China

Tel. +86-791-8396-9553

Email: Xiaoyongzhang1980@gmail.com 


\begin{abstract}
Preparation and biomedical applications of aggregation induced emission (AIE) dye based fluorescent nanoprobes (FNPs) have been extensively investigated in recently years. And great efforts have been devoted toward fabrication of AIE dye based FNPs through both noncovalent and covalent strategies. However, to the best of our knowledge, the preparation of AIE dye based FNPs via dynamic bonds is rarely reported. In this work, ultrabright FNPs based on AIE dyes have been fabricated using aldehyde-terminated polyethylene glycol (CHO-PEG-CHO) with molecular weight of 2000 Da and 2,2'-diaminotetraphenyl ethylene (TPE- $\left.\mathrm{NH}_{2}\right)$ through formation of Schiff base. The resulting TPE contained copolymers are prone to self-assemble into stabilized core shell nanoparticles (TPE@PEG FNPs). The successful formation of TPE@PEG FNPs was confirmed by ${ }^{1} \mathrm{H}$ nuclear magnetic resonance spectroscopy, transmission electron microscopy, fluorescence spectroscopy and fourier transform infrared spectroscopy. Due to their amphipihlic properties, TPE@PEG FNPs displayed high water dispersibility and strong fluorescence intensity. Furthermore, biological evaluation results demonstrated that TPE@PEG FNPs possess excellent biocompatibility and can be facilely internalized by cells. The extraordinary fluorescent properties, excellent water dispersibility as well as the desirable biocompatibility make TPE@PEG FNPs promising candidates for biomedical applications. On the other hand, because of the pH stimuli responsiveness of Schiff base, TPE@PEG FNPs are expected to exhibit some advantages for biomedical applications as compared with the FNPs fabricated by noncovalent and covalent strategies.
\end{abstract}

Keywords: Biological imaging, dynamic bonds, Schiff base, AIE-active nanoprobes 


\section{Introduction}

In the past few decades, the research of fluorescent nanoprobes (FNPs) has attracted numerous attentions because of their broad application prospects in many fields ranging from chemsensors, biomedical and bioimaging. ${ }^{1-11}$ As we all know, one of the most important reasons for the extensive application of FNPs is that the facile establishment of fluorescence labeling methodology for tracing biological species and procedure in living systems and bioimaging. Inspiring by this, an increasing number of FNPs have been drastically exploited and effectively applied in many fields such as biological probes, bioanalysis and cell imaging. Numerous FNPs based on the fluorescent proteins, ${ }^{12-17}$ rare earth-doped up conversion nanoparticles, ${ }^{18-21}$ semiconductor quantum dots, ${ }^{22-29}$ carbon dots ${ }^{30-34}$ and organic dyes ${ }^{35-37}$ have been fabricated and drastically investigated for biomedical application. However, some inherent drawbacks include well-known high cytotoxicity of rare earth-doped nanoparticles resulted by their accumulation in the reticuloendothelial system, difficult synthesis, non-biodegrabradability and non-functionalized hydrophilic entities are appeared in the aforementioned fluorescent materials, which will largely prevent their wide application in biomedical fields. ${ }^{38-41}$ Although the disadvantages of toxicity and poor biocompatibility of inorganic FNPs, were efficiently overcome by fluorescent proteins. The usage of fluorescent proteins has been still seriously limited because of their high cost, low molar absorptivity and low photobleaching. ${ }^{42-44}$ Therefore, fabrication of novel FNPs with low cost, non-toxic and great biodegradable is especially important.

Due to their designability, biocompatibility and biodegradable potential, FNPs based on organic dyes have recently attracted much attentions for biomedical applications. ${ }^{45-47}$ The general strategy for fabrication of FNPs is encapsulation organic dyes into amphiphilic copolymers or covalent conjugation of organic dyes with hydrophilic polymers. These organic dyes contained copolymers exihibited amphipihlic properties and can self assemble into core shell FNPs. The hydrophobic organic dyes are aggregated in the core and the hydrophilic components are coated on the hydrophobic core and served as the shell. All these organic dyes can be facilely converted into water dispersible FNPs. Unfortunately, conventional organic dyes suffer from fluorescence quenching phenomenon when they were dispersed in solution at high concentrations or aggregation state. This phenomenon was named as the aggregation-caused quenching (ACQ) effects. ${ }^{48}$ Therefore it is still difficult to obtain utrabright FNPs using conventional organic dyes for the ACQ effects. To overcome this problem, a number of fantastic organic dyes with aggregation induced emission (AIE) properties were developed. These 
types of organic dyes can emit intense fluorescence in their aggregation state while weak or non-fluorescence in good solvents. These organic dyes with AIE properties are expected to elegantly overcome the ACQ effects of conventional organic dyes, thus provide an effective route for fabrication of FNPs with desirable luminescent properties. ${ }^{49}$ To date, increasing attentions have been devoted toward fabrication of AIE nanoprobes based on AIE dyes such as siloles, cyano-substituted diarylethlene, tetraphenylethene and triphenylethene. ${ }^{46-48}$ Various strategies have been proposed for fabricating AIE dye based nanoprobes to resolve the problems of AIE dyes for biomedical applications..$^{50,49,51-54}$ For example, non-covalent encapsulation of AIE dyes into commercial surfactants and synthestic amphipathlic polymers has been demonstrated. ${ }^{51}$ The results suggested the water dispersibility of AIE dyes can be greatly improved by self assembly with these amphiphilic agents. However, the noncovalent encapsulation of dyes may lead to slowly leak dyes from assemblies due to the weak interactions. ${ }^{55}$ On the other hand, Tang and co-workers have demonstrated that insoluble AIE dyes could be facilely encapsulated into the boving serum albumin (BSA) and then cross-linked by glutaraldehyde, resulting in water dispersible and biocompatible AIE dye based FNPs. More importantly, thus obtained fluorescent biomolecule based on AIE dyes have been perfectly applied in cells and animals. Although this high-efficient strategy overcome the problem of poor water solubility of AIE materials, glutaraldehyde is a well known toxicity molecule to living organisms. Furthermore, a series of covalent strategies including RAFT polymerization, ring-opening polymerization and conjugation reaction etc have also been adopted for fabrication of more stable AIE dye based fluorescent nanoprobes. ${ }^{56-58}$ However, to the best of our knowledge, the fabrication of AIE based nanoprobes via formation of dynamic bonds is seldom been reported.

Schiff base is a very important dynamic bond, that can response to the $\mathrm{pH}$ change and has been widely utilized for fabrication of $\mathrm{pH}$ responsive systems for biomedical applications. In this contribution, an efficient strategy is reported for the first time to fabricate PEGylated AIE dye based nanoprobes via typical Schiff base reaction. The synthetic process could be clearly described in Scheme 1: (i) the aldehyde-terminated polyethylene glycol (PEG) (CHO-PEG-CHO) could be prepared via dehydration procedure between PEG and formylbenzoic acid using DCC/DMAP as dehydrating agent. (ii) The final product (named as TPE@PEG FNPs) can be obtained via Schiff base reaction between synthetic amine-treminated tetraphenyl ethylene (labeled as TPE- $\mathrm{NH}_{2}$ ) and CHO-PEG-CHO. The resulting TPE@PEG FNPs show high water solubility and strong blue-green luminescence. The 
biocompatibility and cell uptake behavior of the obtained TPE@PEG FNPs were further determined.

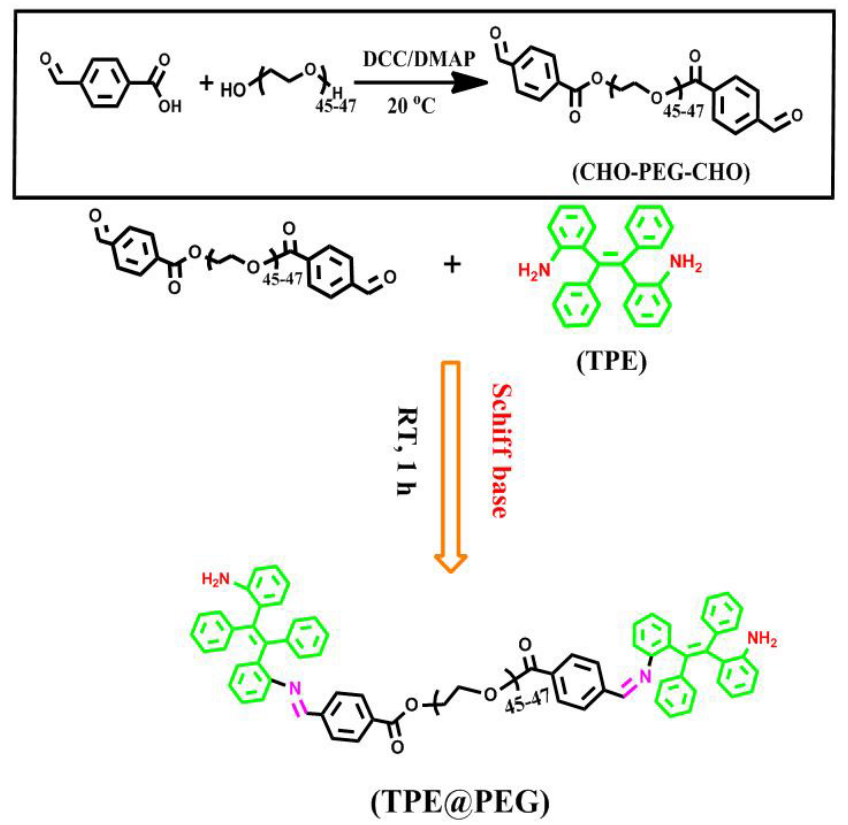

Scheme 1. Synthetic route of CHO-PEG-CHO and preparation of was described in this picture. The TPE- $\mathrm{NH}_{2}$ materials were synthesized by McMurry reaction, which suggesting strong green fluorescence at aggregated state. After TPE- $\mathrm{NH}_{2}$ was covalently conjugated onto the PEG via typical Schiff base reaction, thus obtained TPE-PEG FNPs could be well dispersed in aqueous solution and emit strong green light under stimulated at $365 \mathrm{~nm}$.

\section{Experiment}

\subsection{Materials and Characterization}

All chemicals were of analytical grade and were used as received without any further purification. PEG $(\mathrm{MW}=2000), \quad \mathrm{N}, \mathrm{N}^{\prime}$-Dicyclohexylcarbodiimide $\quad($ DCC, $\quad \mathrm{MW}=206.33, \quad 99.0 \%)$, 4-Dimethylaminopyridine (DMAP, MW = 122.17, 99.0\%) and 4-Carboxybenzaldehyde $(\mathrm{MW}=$ 150.13, 99.8\%) were purchased from Aladdin company (Shanghai, China). Zinc powders, titanium tetrachloride, 2-Aminobenzophenone (MW = 197.23, 99.0\%) and anhydrous tetrahydrofuran (THF) were provided from Heowns (Tianjin, China).

${ }^{1} \mathrm{H}$ nuclear magnetic resonance spectroscopy (NMR) spectra were recorded on Bruker Avance-400 spectrometer with $\mathrm{D}_{2} \mathrm{O}$ as the solvent. The synthetic polymers and materials were characterized by Fourier transform infrared spectroscopy (FT-IR) using $\mathrm{KBr}$ pellets, The Fourier transform infrared 
(FT-IR) spectra were supplied from Nicolet5700 (Thermo Nicolet corporation). Transmission electron microscopy (TEM) images were recorded on a Hitachi 7650B microscope operated at $80 \mathrm{kV}$, the TEM specimens were got by putting a drop of the nanoparticle ethanol suspension on a carbon-coated copper grid.

\subsection{Synthesis of TPE-NH}

The TPE- $\mathrm{NH}_{2}$ was synthesized according to the literature stratrgy. ${ }^{59}$ A suspension of Zn powder (3.57 g, $0.06 \mathrm{moL})$ in dry THF $(40 \mathrm{~mL})$ was drastically stirring at ice-water bath about $10 \mathrm{~min} . \mathrm{TiCl}_{4}(4 \mathrm{~mL})$ was gradually injected into the above-mentioned solution over $30 \mathrm{~min}$. After then, the ice-water bath was removed and the reaction system was heated under reflux for 2 h. After finishing that, the 2-Aminobenzophenone (2.7 g, $0.0137 \mathrm{moL}$ ) dissolved in the dry THF (20 mL) was gradually dropwise added into the reaction system and refluxed overnight. The reaction system was quenched with 5\% aqueous solution of ammonium and extracted with ethylacetate. The obtained organic layer was dried with anhydrous $\mathrm{MgSO}_{4}$ over-night, suction filtration, concentrated using a rotary evaporation. The crude product was recrystallization using n-hexane/diethyl ether and filtered to yield a weak yellow solid. CDCl3; $\delta=4.7-5.0$ ppm, d (4H); $\delta=6.6-6.7$ ppm, m(4H); $\delta=6.9-7.0$ ppm, m(4H); $\delta=7.0-7.15$ ppm, $\mathrm{m}(4 \mathrm{H}) ; \delta=7.4-7.6 \mathrm{ppm}, \mathrm{m}(2 \mathrm{H}) ; \delta=7.9-8.0 \mathrm{ppm}, \mathrm{d}(2 \mathrm{H})$

\subsection{Preparation of CHO-PEG-CHO}

The CHO-PEG-CHO samples were facilely prepared between PEG and 4-Carboxybenzaldehyde using DCC/DMAP system. PEG with molecular weights of 2000 Da (6.52 g, 3.26 mol), 4-Carboxybenzaldehyde (1.96 g, $13.04 \mathrm{mM}$ ) and DMAP (100 mg) were completely dissolved in the anhydrous THF (20 mL) and heated to the $20^{\circ} \mathrm{C}$. Afterwards, the calculated amounts of DCC (3.36 g, $16.3 \mathrm{mM}$ ) in $10 \mathrm{~mL}$ dry THF were injected into aforementioned reaction system in nitrogen atmosphere and stirred for $18 \mathrm{~h}$. When the white solid was filtered. The white CHO-PEG-CHO could be obtained after repeated dissolution in the THF and precipitation in the diethyl ether.

\subsection{Fabrication of TPE@PEG FNPs}

The high water-soluble fluorescent probe was mildly fabricated using CHO-PEG-CHO and TPE- $\mathrm{NH}_{2}$ as precursors via Schiff base reaction. CHO-PEG-CHO (0.18 mM, $400 \mathrm{mg})$ and TPE-NH $2(0.5 \mathrm{mM}$, $180 \mathrm{mg})$ were respectively dissolved in distilled water $(10 \mathrm{~mL})$ and THF $(10 \mathrm{~mL})$ and acutely stirred at 
room temperature for $2 \mathrm{~h}$. The pure product could be obtained via precipitating in anhydrous ether after completely removing water existed in the reaction system using rotary evaporated.

\subsection{Cytotoxicity evaluation of PEG@TPE FNPs}

The cell viability of TPE@PEG FNPs for HeLa cells was evaluated by cell counting kit-8 (CCK-8) assay. Briefly, cells were put into 96-well microplates at a density of $5 \times 10^{4}$ cells $\mathrm{mL}^{-1}$ in $160 \mu \mathrm{L}$ of respective media containing 10\% fetal bovine serum (FBS). After $24 \mathrm{~h}$ of cell attachment, the cells were incubated with $20 \mu \mathrm{g} \mathrm{mL} \mathrm{g}^{-1}$ FNPs for 12 and $24 \mathrm{~h}$. Then nanoparticles were removed and cells were washed with PBS three times. $10 \mu \mathrm{L}$ of CCK-8 dye and $100 \mu \mathrm{L}$ of DMEM cell culture medium were added to each well and incubated for $2 \mathrm{~h}$ at $37^{\circ} \mathrm{C}$. Afterward, plates were analyzed using a microplate reader (VictorШ, Perkin-Elmer). Measurements of formazan dye absorbance were carried out at $450 \mathrm{~nm}$, with the reference wavelength at $620 \mathrm{~nm}$. The values were proportional to the number of live cells. The percent reduction of CCK-8 dye was compared to controls (cells not be exposured to hydrophilic FNPs), which represented 100\% CCK-8 reduction. Three replicate wells were used per microplate, and the experiment was operated for three times. Cell survival was expressed as absorbance relative to that of untreated controls. Results are presented as mean \pm standard deviation (SD).

\subsection{Confocal microscopic imaging}

HeLa cells were cultured in Dulbecco's modified eagle medium (DMEM) supplemented with 10\% heat-inactivated FBS, $2 \mathrm{mM}$ glutamine, $100 \mathrm{U} \mathrm{mL}^{-1}$ penicillin, and $100 \mu \mathrm{g} \mathrm{mL}^{-1}$ of streptomycin. Cell culture was controlled at $37{ }^{\circ} \mathrm{C}$ in a similar humidified condition of $95 \%$ air and $5 \% \mathrm{CO}_{2}$ in culture medium. Culture medium should be updated every three days for maintaining the exponential growth of the cells. Before treatment, cells were seeded in a glass bottom dish with a density of $1 \times 10^{5}$ cells per dish. On the day of treatment, the cells were cultured with TPE@PEG FNPs at a final concentration of $20 \mu \mathrm{g} \mathrm{mL} \mathrm{L}^{-1}$ for $3 \mathrm{~h}$ at $37^{\circ} \mathrm{C}$. Afterward, the cells were washed three times with PBS to remove the TPE@PEG FNPs and then fixed with 4\% paraformaldehyde for $10 \mathrm{~min}$ at room temperature. Cell images were obtained using CLSM Zesis 710 3-channel (Zesis, Germany) with the excitation wavelength of $405 \mathrm{~nm}$.

\section{Results and discussion}

Fabrication of water dispersible, biocompatible and ultrabright FNPs has been the subject of great 
research interest. It has been demonstrated that incorporation of AIE dyes into polymers is a general route to obtain these ultrabright FNPs for the unique AIE properties of these dyes. Although great efforts have been made in fabrication of AIE dye based FNPs through both noncovalent and covalent strategies. The fabrication of AIE dye based FNPs through dynamic bonds is seldom demonstrated. In this work, we reported that AIE dye based FNPs can be facilely fabricated via formation of Schiff base between the aldehyde groups of PEG and the amino groups of TPE- $\mathrm{NH}_{2}$. And the CHO-PEG-CHO was facilely synthesized via dehydration between PEG and 4-Carboxybenzaldehyde using DCC/DMAP system. In order to obtain the dry PEG, PEG was vacuum distillated with dry toluene for three times. The TPE- $\mathrm{NH}_{2}$ could be synthesized using 2-Aminobenzophenone as a precursor according to our previous report. $^{59}$ The structure of synthetic functionalized CHO-PEG-CHO and TPE@PEG could be characterized and confirmed by a series of characterization measurements such as ${ }^{1} \mathrm{H}$ NMR, FT-IR, TEM and so on. Finally, the results of cell uptake experiments demonstrated that TPE-PEG FNPs are promising for biomedical applications.

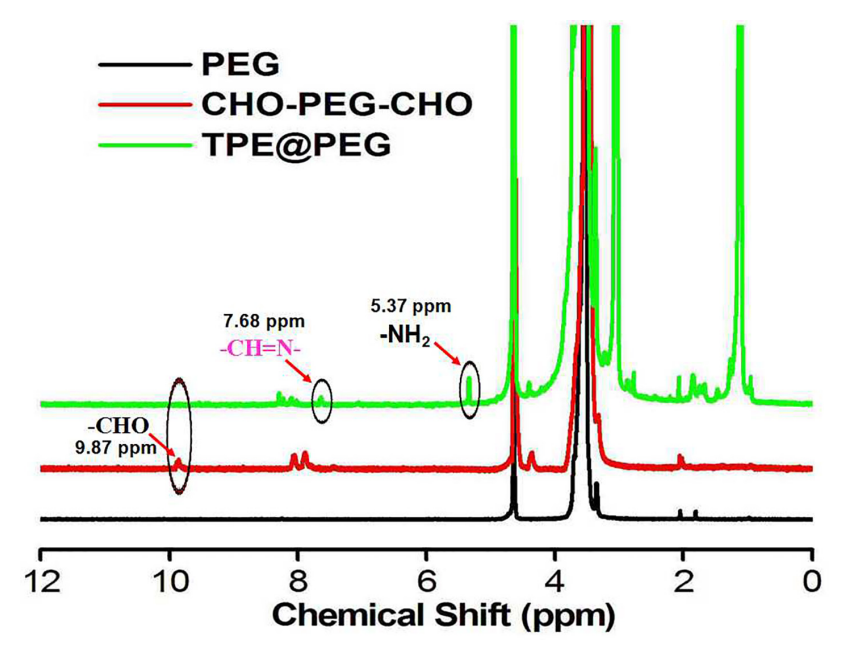

Fig. 1 The ${ }^{1} \mathrm{H}$ NMR spectra of PEG, aldehyde PEG and TPE@PEG samples were measured using $\mathrm{D}_{2} \mathrm{O}$ as solvent, which demonstrate successful preparation of high water-dispersibility FNPs based on TPE.

The structure of PEG, CHO-PEG-CHO, TPE@PEG copolymers could be confirmed using ${ }^{1} \mathrm{H}$ NMR spectra. As shown in Fig. 1, the chemical shift of PEG can be described as following: $\delta=3.5-4.0 \mathrm{ppm}$ $\left(-\mathrm{CH}_{2}-\right), \delta=1.5-2.1 \mathrm{ppm}(-\mathrm{OH})$. After modification with 4-Carboxybenzaldehyde, some new characterization peaks are appeared in the spectrum of CHO-PEG-CHO. The multiplet appeared at $\delta=$ 
7.5-8.5 ppm could be attributed to the introduction of benzene. The single peak at $\delta=9.87 \mathrm{ppm}$ was belonged to the -CHO. More importantly, chemical signal about hydroxyl groups ( $\delta=1.5-2.1 \mathrm{ppm}$ ) of PEG was disappeared in the samples of CHO-PEG-CHO. These results provide powerful evidence that successful introduction of aldehyde group to the two sides of PEG. However, after formation of FNPs between the CHO-PEG-CHO and AIE dye (TPE- $\mathrm{NH}_{2}$ ), novel chemical signals were found in the samples of TPE@PEG copolymers. For example, apart from the signals about PEG, $\delta=7.68$ ppm, $\delta=$ $5.37 \mathrm{ppm}$ and $\delta=3-4 \mathrm{ppm}$ envidenced the existence of $-\mathrm{CH}=\mathrm{N}-,-\mathrm{NH}_{2}$ and $-\mathrm{CH}_{2}$ in the TPE@PEG copolymers. Furthermore, as seen from the red line, the disappearance of peak at $9.87 \mathrm{ppm}$ could be ascribed that successful reaction between $-\mathrm{CHO}$ and $-\mathrm{NH}_{2}$ via Schiff base reaction. These results confirmed that successful formation of Schiff base between CHO-PEG-CHO and TPE- $\mathrm{NH}_{2}$. From the results of typical ${ }^{1} \mathrm{H}$ NMR spectra, we preliminary confirmed that successful fabrication of TPE@PEG FNPs via formation of Schiff base.

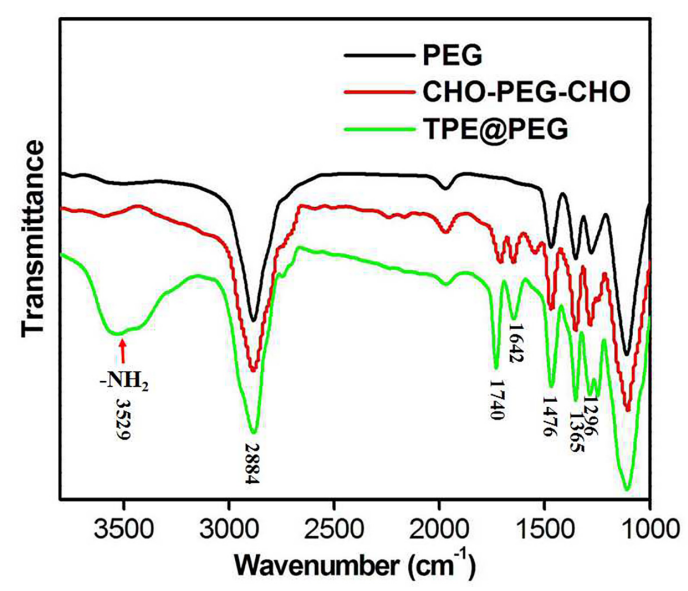

Fig. 2 FT-IR spectra of PEG, CHO-PEG-CHO and TPE@PEG FNPs. The specific adsorption peaks located at 1113 and $2884 \mathrm{~cm}^{-1}$ can be attributed to the C-O and C-H bonds of PEG. After formation of CHO-PEG-CHO, three new peaks located between $1750-1450 \mathrm{~cm}^{-1}$ were emerged, that can be ascribed to the stretching vibration of aromatic ring of benzaldehyde and the $\mathrm{C}=\mathrm{O}$ of ester bond. Furthermore, the stretching vibration of amino groups located at $3529 \mathrm{~cm}^{-1}$ was appeared in the sample of TPE@PEG, indicating that the TPE- $\mathrm{NH}_{2}$ were reacted with benzaldehyde of CHO-PEG-CHO via formation of Schiff base. All of these above results demonstrated that we can fabricate AIE dye based nanoprobes through dynamic bonds.

The FT-IR spectra of PEG, CHO-PEG-CHO and TPE@PEG FNPs were displayed in Fig. 2. A 
characterized peak located at $1113 \mathrm{~cm}^{-1}$ could be attributed to the flexible vibration of C-O from the pure PEG. The absorption peak located around $2884 \mathrm{~cm}^{-1}$ can be assigned to the $\mathrm{C}-\mathrm{H}$ bond of $\mathrm{CH}_{2}$ and $\mathrm{CH}_{3}$ of PEG. As compared with PEG, two new characterized peaks appeared between $1750-1450 \mathrm{~cm}^{-1}$ were emerged in CHO-PEG-CHO. The peak located at $1720 \mathrm{~cm}^{-1}$ can be ascribed to the flexible vibration of $\mathrm{O}-\mathrm{C}=\mathrm{O}$, and the other characterized peak at $1642 \mathrm{~cm}^{-1}$ could be served as vibration of - $\mathrm{CHO}$ and the stretching vibration of aromatic rings, which providing direct evidence that successful conjugation between PEG and 4-Carboxybenzaldehyde based on DCC/DMAP system. After formation of FNPs with AIE dyes (TPE- $\mathrm{NH}_{2}$ ) based on the formylated PEG, the appearance of new incisive special peak at $1740 \mathrm{~cm}^{-1}$ could be ascribed to the formation of $-\mathrm{CH}=\mathrm{N}$ - bonds. Furthermore, an obvious characterized peak appeared at $3529 \mathrm{~cm}^{-1}$ could be thought as vibration of $-\mathrm{NH}_{2}$. These results demonstrate that successful formation of high water-solubility FNPs via typical Schiff base reaction. In summary, we can preliminary draw a conclusion that AIE dye based FNPs can be facilely fabricated via formation of Schiff base based on FT-IR spectral data.

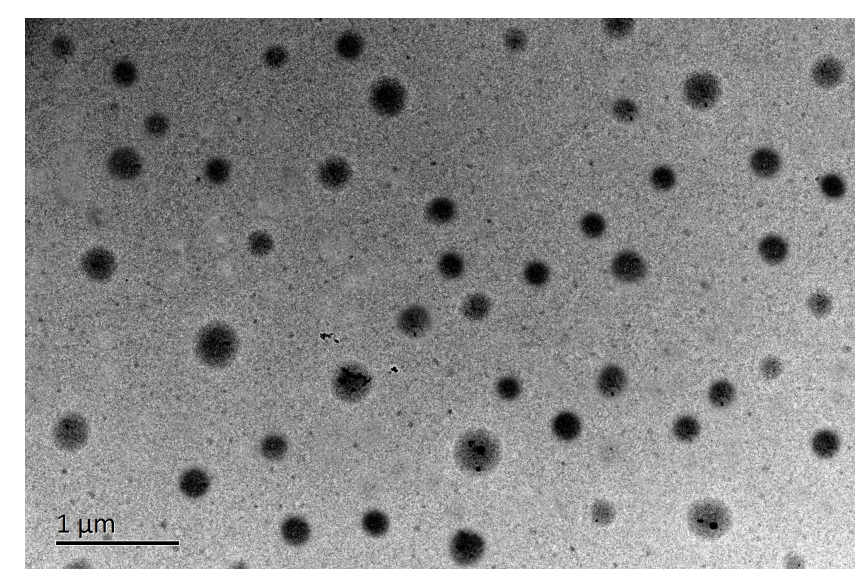

Fig. 3 The representative TEM image of TPE@PEG FNPs. Many spherical nanoparticles with diameter about a few hundred of nanometers have been formed in their aggregated state. The successful formation of nanoparticles implied that amphiphilic TPE@PEG copolymers have synthesized through formation of Schiff base.

The microstructure of TPE@PEG FNPs was characterized by TEM characterization. As shown in Fig. 3, many spherical nanoparticles with diameter of a few hundred nanometers can be observed. Furthermore, the TEM picture provides direct evidence that PEGylated TPE was self assembled into the spherical nanoparticles when they were aggregated in solid state. The reason for the formation of 
spherical nanoparticles by TPE@PEG copolymers could be explained by the formation of amphiphilic copolymers, in which, the hydrophobic AIE dye $\left(\mathrm{TPE}-\mathrm{NH}_{2}\right)$ was encapsulated in the core of TPE@PEG copolymers. While the hydrophilic PEG was covered the surface of AIE core, that renders the excellent water dispersibility of TPE@PEG FNPs. On the other hand, uniform spherical nanoparticles with diameter ranged from100-300 nm can be observed by TEM images. The size of TPE@PEG FNPs calculated based on the TEM images is $185 \pm 21.5 \mathrm{~nm}$. Furthermore, the size distribution of these TPE@PEG FNPs in water was further determined by dynamic light scattering (DLS) measurement. As indicated by the DLS results, the hydrodynamic size distribution of TPE@PEG FNPs was $255.3 \pm 14.4$ nm (Fig. S1). As compared with the TEM resutls, the size of TPE@PEG FNPs is relative large from the DLS measurement. The different size distribution of TPE@PEG FNPs might be ascribed to the shrinkage of micelles for TEM characterization. As compared with the size of cells, the size of TPE@PEG FNPs is very suitable for cell uptaken. Therefore, these TPE@PEG FNPs are suitable for biological imaging and fabrication of nanotheranostics.
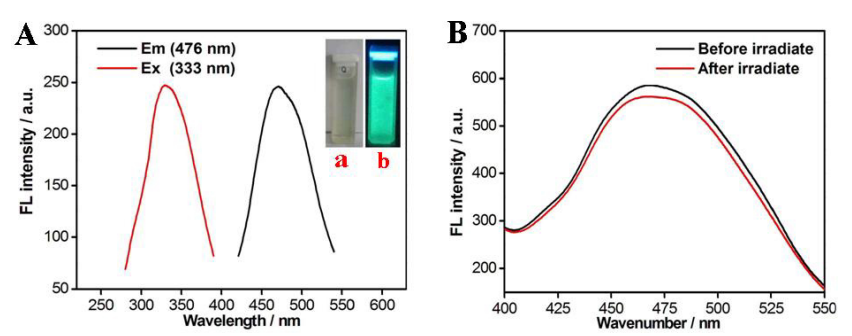

Fig. 4 (A) The excitation and emission PL spectra of TPE@PEG FNPs. 5 mg TPE@PEG materials was dispersed in $20 \mathrm{~mL}$ water, which could serve as the standard solution to detect their maximum excitation and emission wavelength, and the result of PL spectra demonstrated that excitation and emission wavelength of TPE@PEG water solution were respectively located at $333 \mathrm{~nm}$ and $476 \mathrm{~nm}$. Inset images are the TPE@PEG FNPs dispersed in pure water (a: left cuvette was not irradiated by UV lamp) and (b: right cuvette was irradiated by UV lamp at $\lambda=365 \mathrm{~nm}$ ). (B) The FL spectra of TPE@PEG FNPs dispersed in pure water for evaluating their photostability. The TPE@PEG FNPs solution was continuously irradiated by UV lamp at $365 \mathrm{~nm}$ for $1 \mathrm{~h}$.

The successful formation of amphiphilic FNPs was also evidenced by the UV-Vis spectrum. The entire spectrum of TPE@PEG suspension began to increase when wavelength around $400 \mathrm{~nm}$ with a 
small peak located at $350 \mathrm{~nm}$, implying that TPE@PEG copolymers were self assembled into nanoparticles in aqueous solution. These well dispersed TPE@PEG FNPs can effectively enhance the light absorption but avoid light transmission (Fig. S2), indicating the good dispersibility of TPE@PEG FNPs in aqueous solution. The above results clearly demonstrated that successful formation of Schiff base between TPE- $\mathrm{NH}_{2}$ and CHO-PEG-CHO, leading to the amphiphilic properties of TPE@PEG copolymers. On the other hand, due to their amphiphilic properties, TPE@PEG copolymers can be well dispersed in pure water solution. Uniform water solution can be observed when TPE@PEG copolymers were dispersed in water in the absent of any organic solvents (left cuvette of Fig. 4A). Even the suspension was deposited for more than $24 \mathrm{~h}$, no noticeable precipitation was observed. It is well known that TPE molecule is an excellent dye with remarkable AIE properties. Therefore, the TPE@PEG suspension is expected to be high luminescent in aqeuos solution due to the aggregation of TPE molecule. Herein, we observed very strong fluorescence when TPE@PEG aqueous solution was irradiated by UV lamp at $\lambda=365 \mathrm{~nm}$ (right cuvette of Fig. 4A). These results implied that TPE-NH has been successfully encapsulated by PEG. And the hydrophobic dye TPE- $\mathrm{NH}_{2}$ was aggregated as the core, while the hydrophilic PEG was coated on the hydrophobic core as the hydrophilic shell. The detailed fluorescent properties of TPE@PEG was determined by fluorescent spectroscopy. As shown in Fig. 4A, the maximum emission wavelength of TPE@PEG FNPs was located around $473 \mathrm{~nm}$ with maxmium excitation peak at $333 \mathrm{~nm}$. The quantum yield (QY) of fluorescent nanoprobes is an important property of their biomedical applications. Therefore, the QY of TPE@PEG FNPs was measured using quinine sulfate as the reference dye. We demonstrated that QY of TPE@PEG FNPs is as high as $25.4 \%$. Furthermore, the photostability was also determined by continuous irradiation the TPE@PEG suspension with UV lamp at 365 nm for 1 h. The fluorescent intensity of TPE@PEG FNPs is about 588.5. After UV lamp irradiation, the fluorescent intensity is still geater than 561.9. This suggested that TPE@PEG FNPs have excellent photostability. The pH responsiveness of TPE@PEG FNPs was also investigated. As shown in Fig. S3, TPE@PEG FNPs become turbid when the pH value was decreased to 5 , indicating that large agglomerates were formed at $\mathrm{pH}=5$. The formation of agglomerates was also clearly observed when TPE@PEG FNPs were irradiated with UV lamp. The formation of agglomerates suggested that the dissociation of PEG and TPE. The pH responsiveness of TPE@PEG FNPs implied that these luminescent nanoprobes were potentially utilized for stimuli responsive drug delivery carries. Therefore, we could conclue that TPE@PEG FNPs possess excellent 
water dispersibility, remarkable fluorescent properties and $\mathrm{pH}$ responsiveness. And these properties made TPE@PEG FNPs very promising candidates for biomedical applications.

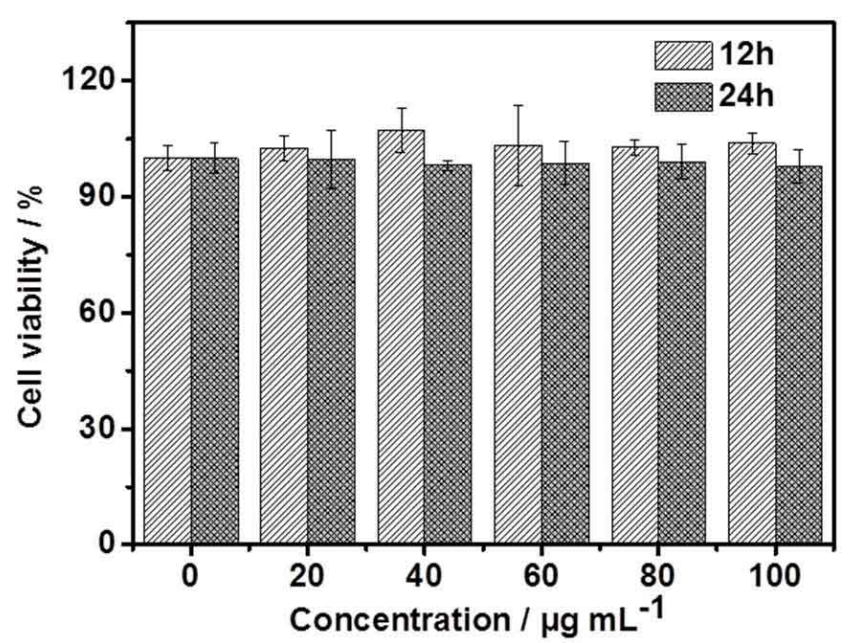

Fig. 5 Biocompatibility evaluation of TPE@PEG FNPs. HeLa cells were incubated with different concentrations (20-100 $\mu \mathrm{g} \mathrm{mL}{ }^{-1}$ ) of TPE@PEG FNPs for 12 and 24 h. The cell viability of TPE@PEG FNPs was determined using CCK-8 assay. It can be seen that the cell viability of TPE@PEG FNPs is still greater than 92\% even the concentration of TPE@PEG FNPs is as high as $100 \mu \mathrm{g} \mathrm{mL}^{-1}$. These results demonstrated the good biocompatibility of TPE@PEG FNPs.

The biocompatibility of TPE@PEG FNPs was further determined using CCK-8 assay. ${ }^{60-63}$ Cells were incubated with 20-100 $\mu \mathrm{g} \mathrm{mL} \mathrm{m}^{-1}$ of TPE@PEG FNPs for 12 and $24 \mathrm{~h}$. As compared with the control cells, the cell viability values of TPE@PEG FNPs showed no significant change when cells were incubated with different concentrations of TPE@PEG FNPs. Even the concentration of TPE@PEG FNPs is as high as $100 \mu \mathrm{gL}^{-1}$, the cell viability value is still greater than $92 \%$ after $12 \mathrm{~h}$ incubation. Furthermore, no cytotoxic effects were observed even the incubation time was increased to 24 h. Due to the internalization of TPE@PEG FNPs by cells, the cell viability values of nanoparticles treated cells are even greater than that of control cells. The excellent biocompatibility provide great potential of TPE@PEG FNPs for bioimaging applications. 

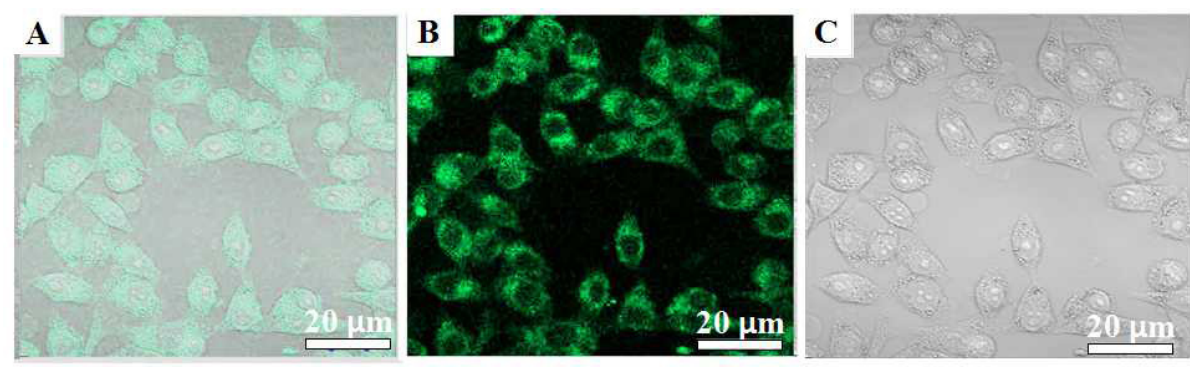

Fig. 6 CLSM images of Hela cells incubated with $20 \mu \mathrm{g} \mathrm{mL} \mathrm{m}^{-1}$ of great water-solubility FNPs for $3 \mathrm{~h}$. (A) merged images of (B) and (C), (B) cells were excited with a 405 nm laser, (C) Bright fields. Scale bar $=20 \mu \mathrm{m}$.

Based on the biocompatibility results, the cell uptake behavior of TPE@PEG FNPs was further investigated. Fig. 6 displayed the CLSM images of HeLa cells when they were incubated with $20 \mu g$ $\mathrm{mL}^{-1}$ of TPE@PEG FNPs for 3 h. The bright field image of cells was shown in Fig. 6A. It can been seen that the cells still kept their normal morphology. This is well consistent with the cell viability results, and further confirmed the good biocompatibility of TPE@PEG FNPs. After the cells were irradiated by a laser with wavelength $405 \mathrm{~nm}$, strong fluorescent signal can be observed (Fig. 6B). And the cell morphology can be also outlined by mapping the fluorescent signal. We found that many dark areas were surrounded by green fluorescence. These dark areas should be the location of the cell nucleus. These results demonstrated that TPE@PEG FNPs could be internalized by HeLa cells and mainly distributed in cytoplasm. The concentration of TPE@PEG FNPs used for cell imaging is $20 \mu \mathrm{g}$ $\mathrm{mL}^{-1}$, which is much lower the maxium concentration $\left(100 \mu \mathrm{g} \mathrm{mL} \mathrm{L}^{-1}\right)$ of for biocompatibility evaluation. Therefore, TPE@PEG FNPs are biocompatible enough for bioimaging applications. The fabrication of AIE dye based FNPs has recently attracted great research interest due to the unique AIE properties, which could elegantly overcome the ACQ effects of conventional organic dyes. Previously, both noncovalent and covalent strategies have been demonstrated for the preparation of AIE FNPs for bioimaging applications. ${ }^{49}$ However, the fabrication of AIE dye based FNPs through dynamic bonds has rarely reported. In this work, we reported a rather facile method for preparation of AIE dye based FNPs via formation of Schiff base between the amino groups contained AIE dye (TPE- $\left.\mathrm{NH}_{2}\right)$ and the CHO-PEG-CHO. The reaction just need mix the TPE- $\mathrm{NH}_{2}$ and CHO-PEG-CHO at room temperature, under air atmoshpere within $1 \mathrm{~h}$, and without requirement of catalysts. It is rather simple, fast, effective and suitable for preparation of AIE dye based FNPs at large scale. Combination of their excellent 
fluorescent properties, high water dispersibility, suitable size and desirable biocompatibility, the TPE@PEG FNPs should be of great potential for biomedical applications. Furhtermore, the TPE@PEG FNPs should also be important candidates for fabrication of multifunctional and stimuli responsive nanotherapeutic systems due to the existence of $\mathrm{pH}$ responisve Schiff base.

\section{Conclusion}

In summary, a facile method for preparation of AIE dye based fluorescent nanoprobes was reported via typical Schiff reaction using CHO-PEG-CHO as the hydrophilic component and amino-terminated TPE dyes as the hydrophobic component for the first time. The obtained copolymers showed amphipihlic properties and are readily self assembled into fluorescent nanoparticles with diameter about $200 \mathrm{~nm}$. The TPE@PEG FNPs emitted strong fluorescence, displayed excellent water dispersibility, good biocompatibility, making them promising for biomedical applications. More importantly, the TPE@PEG FONs could be further conjugated with other components such as drugs and targeting agents by take full advantages of functional groups such as amino groups, which provide great potential for the fabrication of multifunctional nanotherapeutic systems based on the TPE@PEG FNPs. Finally, due to its simpilicity, effectiveness and scaleable potential, this strategy described in this work are also useful for fabrication of many other $\mathrm{pH}$ responsive AIE dye based FNPs. Some other related studies succeed in this work were carried out in our lab.

\section{Acknowledgements}

This research was supported by the National Science Foundation of China (Nos. 21134004, 21201108, 51363016, 21474057, 21564006, 21561022), and the National 973 Project (Nos. 2011CB935700).

\section{References}

1. Qin, A.; Lam, J. W.; Tang, B. Z. Prog. Polym. Sci. 2012, 37, 182-209.

2. $\quad$ Zhao, Q.; Li, K.; Chen, S.; Qin, A.; Ding, D.; Zhang, S.; Liu, Y.; Liu, B.; Sun, J. Z.; Tang, B. Z. J. Mater. Chem. 2012, 22, 15128-15135.

3. Zhang, L.; Wang, E. Nano Today 2014, 9, 132-157.

4. $\quad$ Liu, M.; Zhang, X.; Yang, B.; Li, Z.; Deng, F.; Yang, Y.; Zhang, X.; Wei, Y. Carbohyd. Polym. 2015, 121, 49-55.

5. Hui, J.; Zhang, X.; Zhang, Z.; Wang, S.; Tao, L.; Wei, Y.; Wang, X. Nanoscale 2012, 4, 6967-6970.

6. Hamilton, G. R.; Sahoo, S. K.; Kamila, S.; Singh, N.; Kaur, N.; Hyland, B. W.; Callan, J. F. Chem. Soc. Rev. 2015.

7. $\quad$ Mérian, J.; Gravier, J.; Navarro, F.; Texier, I. Molecules 2012, 17, 5564-5591. 
8. Yin, J.; Hu, Y.; Yoon, J. Chem. Soc. Rev. 2015.

9. Zhu, L.; Younes, A. H.; Yuan, Z.; Clark, R. J. J. Photoch. Photobio. A 2015, 311, 1-15.

10. Zhu, L.; Yuan, Z.; Simmons, J. T.; Sreenath, K. RSC Adv. 2014, 4, 20398-20440.

11. Liu, M.; Ji, J.; Zhang, X.; Zhang, X.; Yang, B.; Deng, F.; Li, Z.; Wang, K.; Yang, Y.; Wei, Y. J. Mater. Chem. B 2015, 3, 3476-3482.

12. Ha, T.; Tinnefeld, P. Annu. Rev. Phys. Chem. 2012, 63, 595-617.

13. Jin, L.; Han, Z.; Platisa, J.; Wooltorton, J. R.; Cohen, L. B.; Pieribone, V. A. Neuron 2012, 75, 779-785.

14. Kalyanaraman, B.; Darley Usmar, V.; Davies, K. J.; Dennery, P. A.; Forman, H. J.; Grisham, M. B.; Mann, G. E.; Moore, K.; Roberts, L. J.; Ischiropoulos, H. Free Radical Bio. Med. 2012, 52, 1-6.

15. Miyawaki, A. Annu. rev. phys. chem. 2011, 80, 357-373.

16. Zhang, J.; Campbell, R. E.; Ting, A. Y.; Tsien, R. Y. Nat. Rev. Mol. Cell Biology 2002, 3, 906-918.

17. Miesenböck, G.; De Angelis, D. A.; Rothman, J. E. Nature 1998, 394, 192-195.

18. Aigouy, L.; De Wilde, Y.; Mortier, M. Appl. Phys. lett. 2003, 83, 147-149.

19. Schuetz, P.; Caruso, F. Chem. Mater. 2002, 14, 4509-4516.

20. Tian, G.; Gu, Z.; Liu, X.; Zhou, L.; Yin, W.; Yan, L.; Jin, S.; Ren, W.; Xing, G.; Li, S. J. Phys. Chem. C 2011, 115, 23790-23796.

21. Soga, K.; Tokuzen, K.; Tsuji, K.; Yamano, T.; Hyodo, H.; Kishimoto, H. Eur. J. Inorg. Chem. 2010, 2010, 2673-2677.

22. Chan, W. C.; Maxwell, D. J.; Gao, X.; Bailey, R. E.; Han, M.; Nie, S. Curr. Opin. Biotech. 2002, 13, 40-46.

23. Chan, W. C.; Nie, S. Science 1998, 281, 2016-2018.

24. Dong, Y.; Wang, R.; Li, G.; Chen, C.; Chi, Y.; Chen, G. Anal. chem. 2012, 84, 6220-6224.

25. Gao, X.; Yang, L.; Petros, J. A.; Marshall, F. F.; Simons, J. W.; Nie, S. Curr. Opin. Biotech. 2005, 16, 63-72.

26. Gerion, D.; Pinaud, F.; Williams, S. C.; Parak, W. J.; Zanchet, D.; Weiss, S.; Alivisatos, A. P. J. Phys. Chem. B 2001, 105, 8861-8871.

27. Kairdolf, B. A.; Smith, A. M.; Stokes, T. H.; Wang, M. D.; Young, A. N.; Nie, S. Annu. Rev. Anal. Chem. 2013, 6, 143-162.

28. Resch Genger, U.; Grabolle, M.; Cavaliere Jaricot, S.; Nitschke, R.; Nann, T. Nat. Methods 2008, 5, 763-775.

29. Peng, J.; Gao, W.; Gupta, B. K.; Liu, Z.; Romero Aburto, R.; Ge, L.; Song, L.; Alemany, L. B.; Zhan, X.; Gao, G. Nano lett. 2012, 12, 844-849.

30. Du, F.; Zeng, F.; Ming, Y.; Wu, S. Microchim. Acta 2013, 180, 453-460.

31. Zhu, A.; Qu, Q.; Shao, X.; Kong, B.; Tian, Y. Angew. Chem. 2012, 124, 7297-7301.

32. Bhunia, S. K.; Saha, A.; Maity, A. R.; Ray, S. C.; Jana, N. R. Sci. Rep. 2013, 3, Doi:10.1038/srep01473.

33. Zhang, X.; Wang, S.; Liu, M.; Yang, B.; Feng, L.; Ji, Y.; Tao, L.; Wei, Y. Phys. Chem. Chem. Phys. 2013, 15, 19013-19018.

34. Zhang, X.; Wang, S.; Zhu, C.; Liu, M.; Ji, Y.; Feng, L.; Tao, L.; Wei, Y. J. Colloid Interf. Sci. 2013, 397, 39-44.

35. Yin, J.; Kwon, Y.; Kim, D.; Lee, D.; Kim, G.; Hu, Y.; Ryu, J. H.; Yoon, J. J. Am. Chem. Soc. 
2014, 136, 5351-5358.

36. Yuan, L.; Lin, W.; Zheng, K.; He, L.; Huang, W. Chem. Soc. Rev. 2013, 42, 622-661.

37. Zhang, S.; Wu, T.; Fan, J.; Li, Z.; Jiang, N.; Wang, J.; Dou, B.; Sun, S.; Song, F.; Peng, X. Org. Biomol. Chem. 2012, 11, 555-558.

38. Chandra, S.; Das, P.; Bag, S.; Laha, D.; Pramanik, P. Nanoscale 2011, 3, 1533-1540.

39. Ho, Y. P.; Leong, K. W. Nanoscale 2010, 2, 60-68.

40. Wu, X.; He, X.; Wang, K.; Xie, C.; Zhou, B.; Qing, Z. Nanoscale 2010, 2, 2244-2249.

41. Shiohara, A.; Prabakar, S.; Faramus, A.; Hsu, C. Y.; Lai, P.-S.; Northcote, P. T.; Tilley, R. D. Nanoscale 2011, 3, 3364-3370.

42. $\quad$ Cai, Z.; Ye, Z.; Yang, X.; Chang, Y.; Wang, H.; Liu, Y.; Cao, A. Nanoscale 2011, 3, 1974-1976.

43. Day, R. N.; Davidson, M. W. Chem. Soc. Rev. 2009, 38, 2887-2921.

44. Zimmer, M. Chem. Rev. 2002, 102, 759-782.

45. Liu, M.; Zhang, X.; Yang, B.; Deng, F.; Li, Z.; Wei, J.; Zhang, X.; Wei, Y. Appl. Surf. Sci. 2014, 322, 155-161.

46. Liu, M.; Wang, K.; Zhang, X.; Zhang, X.; Li, Z.; Zhang, Q.; Huang, Z.; Wei, Y. Tetrahedron 2015, 71, 5452-5457.

47. Zhang, X.; Zhang, X.; Yang, B.; Wei, Y. Chin. J. Polym. Sci. 2014, Accepted.

48. Zhang, X.; Wang, K.; Liu, M.; Zhang, X.; Tao, L.; Chen, Y.; Wei, Y. Nanoscale 2015, 7, 11486-11508.

49. Zhang, X.; Zhang, X.; Tao, L.; Chi, Z.; Xu, J.; Wei, Y. J. Mater. Chem. B 2014, 2, 4398-4414.

50. Zhang, X.; Liu, M.; Yang, B.; Zhang, X.; Wei, Y. Colloid. Surface. B 2013, 112, 81-86.

51. Zhang, X.; Zhang, X.; Wang, S.; Liu, M.; Tao, L.; Wei, Y. Nanoscale 2013, 5, 147-150.

52. Zhang, X.; Zhang, X.; Wang, S.; Liu, M.; Zhang, Y.; Tao, L.; Wei, Y. ACS Appl. mater. Inter. 2013, 5, 1943-1947.

53. Zhang, X.; Zhang, X.; Yang, B.; Hui, J.; Liu, M.; Chi, Z.; Liu, S.; Xu, J.; Wei, Y. Polym. Chem. 2014, 5, 683-688.

54. Wang, K.; Zhang, X.; Zhang, X.; Yang, B.; Li, Z.; Zhang, Q.; Huang, Z.; Wei, Y. Polym. Chem. 2015, 6, 1360-1366.

55. Lu, H.; Zhao, X.; Tian, W.; Wang, Q.; Shi, J. RSC Adv. 2014, 4, 18460-18466.

56. Zhang, X.; Zhang, X.; Yang, B.; Liu, L.; Deng, F.; Hui, J.; Liu, M.; Chen, Y.; Wei, Y. RSC Adv. 2014, 4, 24189-24193.

57. Zhang, X.; Zhang, X.; Yang, B.; Liu, L.; Hui, J.; Liu, M.; Chen, Y.; Wei, Y. RSC Adv. 2014, 4, 10060-10066.

58. Zhao, Y.; Wu, Y.; Yan, G.; Zhang, K. RSC Adv. 2014, 4, 51194-51200.

59. Wan, Q.; Wang, K.; Du, H.; Huang, H.; Liu, M.; Deng, F.; Dai, Y.; Zhang, X.; Wei, Y. Polym. Chem. 2015, 6, 5288-5294.

60. Zhang, X.; Hu, W.; Li, J.; Tao, L.; Wei, Y. Toxicol. Res. 2012, 1, 62-68.

61. Zhang, X.; Liu, M.; Zhang, X.; Deng, F.; Zhou, C.; Hui, J.; Liu, W.; Wei, Y. Toxicol. Res. 2015, 4, 160-168.

62. Zhang, X.; Qi, H.; Wang, S.; Feng, L.; Ji, Y.; Tao, L.; Li, S.; Wei, Y. Toxicol. Res. 2012, 1, 201-205.

63. Zhang, X.; Wang, S.; Liu, M.; Hui, J.; Yang, B.; Tao, L.; Wei, Y. Toxicol. Res. 2013, 2, 335-346. 


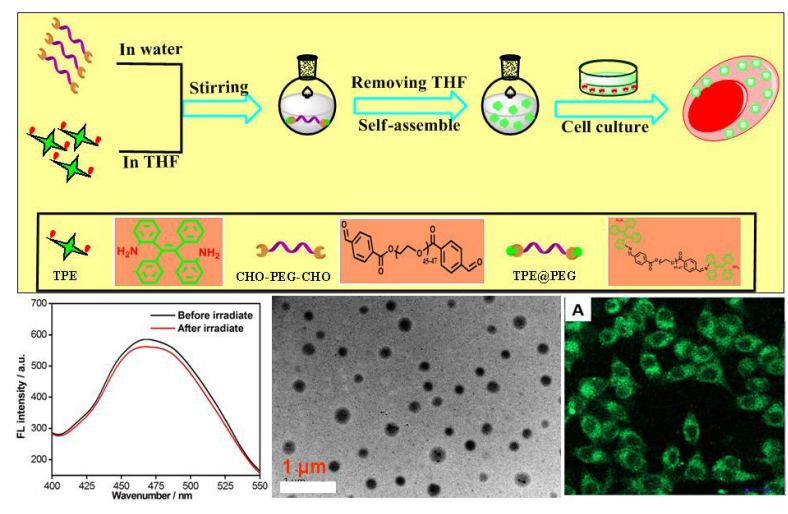

This work reported the fabrication of AIE dye based fluorescent nanoprobes through formation of dynamic bonds, which should be very useful for development of stumuli responsive nanotheranostics. 